\title{
Calculations of Strong Field Multiphoton Processes in Alkali Metal Atoms
}

K. J. Schafer, M. B. Gaarde, K. C. Kulander, B. Sheehy, L. F. DiMauro

This article was submitted to

Multiphoton Processes 1999: $8^{\text {th }}$ International Conference on Multiphoton Processes

Monterey, CA

October 3-8. 1999

U.S. Department of Energy

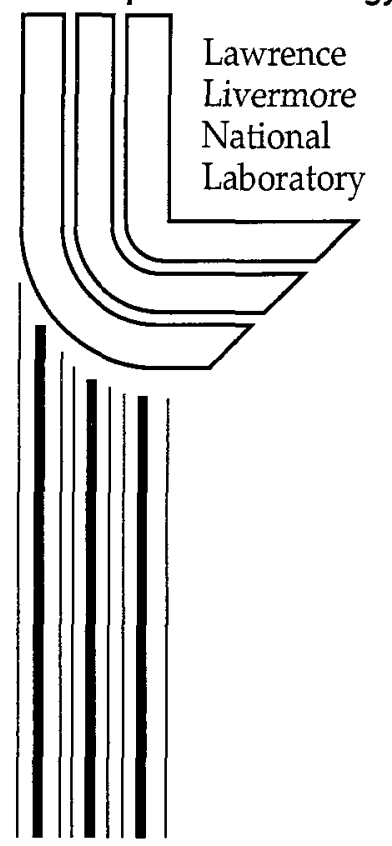

March 15, 2000 


\section{DISCLAIMER}

This document was prepared as an account of work sponsored by an agency of the United States Government. Neither the United States Government nor the University of California nor any of their employees, makes any warranty, express or implied, or assumes any legal liability or responsibility for the accuracy, completeness, or usefulness of any information, apparatus, product, or process disclosed, or represents that its use would not infringe privately owned rights. Reference herein to any specific commercial product, process, or service by trade name, trademark, manufacturer, or otherwise, does not necessarily constitute or imply its endorsement, recommendation, or favoring by the United States Government or the University of California. The views and opinions of authors expressed herein do not necessarily state or reflect those of the United States Government or the University of California, and shall not be used for advertising or product endorsement purposes.

This is a preprint of a paper intended for publication in a journal or proceedings. Since changes may be made before publication, this preprint is made available with the understanding that it will not be cited or reproduced without the permission of the author.

This work was performed under the auspices of the United States Department of Energy by the University of California, Lawrence Livermore National Laboratory under contract No. W-7405-Eng-48.

This report has been reproduced directly from the best available copy.

Available electronically at http://www.doc.gov/bridge

Available for a processing fee to U.S. Department of Energy

And its contractors in paper from

U.S. Department of Energy

Office of Scientific and Technical Information

P.O. Box 62

Oak Ridge, TN 37831-0062

Telephone: (865) 576-8401

Facsimile: (865) 576-5728

E-mail: reports@adonis.osti.gov

Available for the sale to the public from

U.S. Department of Commerce

National Technical Information Service

5285 Port Royal Road

Springfield, VA 22161

Telephone: (800) 553-6847

Facsimile: (703) 605-6900

E-mail: orders@ntis.fedworld.gov

Online ordering: http://www.ntis.gov/ordering.htm

Or

Lawrence Livermore National Laboratory

Technical Information Department's Digital Library

http://www.llnl.gov/tid/Library.html 


\title{
Calculations of Strong Field Multiphoton Processes in Alkali Metal Atoms
}

\author{
K. J. Schafer ${ }^{1}$, M. B. Gaarde ${ }^{2}$, K. C. Kulander ${ }^{3}$, \\ B. Sheehy ${ }^{4}$, and L. F. DiMauro ${ }^{4}$ \\ ${ }^{1}$ Department of Physics and Astronomy, Louisiana State University, Baton Rouge, LA 70803 \\ ${ }^{2}$ Department of Physics, Lund Institute of Technology; P. O. Box 118. S-22100 Lund, Sweden \\ ${ }^{3}$ TAMP Group, Lawrence Livermore National Laboratory, Livermore, CA 94551 \\ ${ }^{4}$ Chemistry Department, Brookhaven National Laboratory, Upton, New York 11973
}

\begin{abstract}
The development of a new class of laser systems, capable of producing intense radiation in the mid-infrared (MIR) regime (photon energies between 0.3 and $0.4 \mathrm{eV}$ ), opens the possibility of observing multiphoton processes in a nerr class of systems with lower ionization potentials than those previously studied. Of particular interest are the alkali metal atoms, which are true one-(valence)-electron systems. We present theoretical calculations of above threshold ionization (ATI) and high harmonic generation (HHG) from alkali metal atoms subject to 3-4 $\mu \mathrm{m}$ laser irradiation. The ATI calculations, which use a multiple gauge propagation method, show a striking dependence in the production of high-order photoelectrons on the electron-ion potential. The HHG calculations illustrate the importance of the strong ground-to-first excited state coupling in multiphoton processes in the alkali metals.
\end{abstract}

\section{INTRODUCTION}

Interest in strong field multiphoton physics continues to flourish, nurtured both by fundamental discoveries and a growing list of applications. Until recently almost all of the available high power lasers provided radiation in the visible/near-infrared region. This has meant that most studies of multiphoton physics have been performed on rather tightly bound systems such as the rare gases [1]. The advent of a new class of laser systems, capable of producing intense $\left(1-2 \mathrm{TW} / \mathrm{cm}^{2}\right)$ radiation in the mid-infrared (MIR) regime (wavelengths between 3 and $4 \mu \mathrm{m}$. photon energies between 0.3 and $0.4 \mathrm{eV}$ ) [2], opens the possibility of observing multiphoton processes in a new group of systems with lower ionization potentials than those previously studied. Of particular interest are the alkali metal atoms which are true one-(valence)-electron systems, and can thus be modeled very accurately within a single-active-electron approximation.

There are many similarities between an alkali metal atom interacting with a strong MIR laser field, and the well-studied case of a rare gas atom subjected to 
a near-IR ( $825 \mathrm{~nm}$ or $1.5 \mathrm{eV}$ ) field. Potassium, for instance, has an ionization potential $\left(I_{p}\right)$ of $4.32 \mathrm{eV}$, corresponding to $\sim 10$ mid-IR laser photons, and argon has an $I_{p}$ of $15.76 \mathrm{eV}$, corresponding to 10-12 near-IR photons. Simple scaling arguments would indicate that the ionization dynamics of potassium interacting with 3-4 $\mu \mathrm{m}$ light at intensities of $10^{12} \mathrm{~W} / \mathrm{cm}^{2}$ should be similar to those of argon interacting with $825 \mathrm{~mm}$ light at intensities of $10^{14} \mathrm{~W} / \mathrm{cm}^{2}$. The Keldysh parameter

$\gamma=\sqrt{T_{p} / 2 U_{p}}[3]$, which compares a free electron's cycle averaged kinetic energy (the ponderomotive energy $\left.U_{p}[4]\right)$ to $I_{p}$, provides a measure of the necessary strength of this interaction. As $\gamma$ becomes less than one the ionization dynamics are dominated by tunneling and are well within the non-perturbative regime. Potassium atoms interacting with a $1 \mathrm{TW} / \mathrm{cm}^{2}, 4 \mu \mathrm{m}$ field will have the same Keldysh parameter, implying similar ionization dynamics, as the well studied case of xenon with 70 $\mathrm{TW} / \mathrm{cm}^{2}, 0.8 \mu \mathrm{m}$ pulses.

Scaling arguments based on any single parameter, however, are only approximately predictive and may overlook important effects. Returning to the comparison between potassium at $3.2 \mu \mathrm{m}$ to argon at $0.8 \mu \mathrm{m}$, we note that the separation between the ground state and the first excited state in terms of the number of photons is a factor of 2 smaller in the alkali metals than it is in the rare gases. There is accordingly a very strong coupling between the two states in the presence of the intense field in the alkali metals which is absent in the rare gases. Our calculations demonstrate that this coupling has a profound effect on the ionization dynamics and therefore on multiphoton processes such as harmonic generation [5].

In this paper we present an account of the theoretical methods that we have used to investigate strong field processes in alkali metal atoms using MIR light. Many of the methods discussed have previously been applied to studies of the rare gases using visible/near-infrared wavelengths [6]. However, the MIR wavelength regime is more demanding computationally than the visible, so we have introduced a multiple gauge propagation method to increase the efficiency of our calculations. We also present sample results from ATI [7] and HHG calculations [5]. A discussion of the first strong field MIR experiments in alkali metal atoms can be found in the paper by Sheehy et al. in this volume [8].

\section{NUMERICAL METHODS}

Our calculations of multiphoton processes in the alkali metals are carried out by direct numerical integration of the time-dependent Schrödinger equation (TDSE) using the single active electron (SAE) approximation [6]. To calculate HHG spectra we use the same methods which have been successfully applied to the rare gases. To make the ATI calculations at MIR wavelengths tractable, we must modify our approach to more efficiently describe the ionized electron wave function. In this section we provide a detailed description of our numerical methods.

We begin by expanding the time-dependent state vector in a mixed basis of discretized radial functions times spherical harmonics, 


$$
|\psi(t)\rangle=\sum_{\ell=0}^{\ell_{\max }} \psi_{\ell}\left(r_{j}, t\right)|\ell\rangle
$$

where $\langle\theta, \phi \mid \ell\rangle=Y_{\ell}^{0}(\theta, \phi)$. Since we restrict ourselves to linear polarization, the $m_{\ell}$ quantum number is conserved. For this reason, the $m_{\ell}$ label is suppressed in the following equations. Atomic units $\left(\hbar=m_{e}=e=1, c \approx 137\right)$ are used throughout.

We use the semi-empirical pseudo-potentials given in [9] to describe the valence electron-ion core interaction. These potentials have three parts, a core potential $V_{c}$, a polarization term $V_{p o l}$, and a $-1 / r$ potential at long range. $V_{c}$ represents the shielding of the nuclear charge by the core electrons, as well as the orthogonality constraints imposed by the exclusion principle. The polarization of the ion charge cloud is accounted for through dipole and quadrupole potentials proportional to $r^{-4}$ and $r^{-6}$ respectively. To obtain the correct energies and transition matrix elements, the short range terms $V_{c}$ and $V_{p o l}$ are in general dependent on the angular momentum channel,

$$
V(\vec{r})=\sum_{\ell} V_{\ell}(r)|\ell\rangle\langle\ell|-1 / r
$$

where $V_{\ell}$ is the sum of the core and polarization terms in a given $\ell$ channel. For $\ell>3$ the potential at small $r$ is dominated by the centrifugal term from the kinetic energy and the short range potentials are irrelevant. Since the pseudopotential is $\ell$-dependent it is non-local. However, the range over which it is non-local is restricted to distances where $V_{c}$ and $V_{p o l}$ are non-zero, i.e., close to the ion core. The potential is effectively local for distances greater than about 5-10 au. As an example, Fig. 1 shows the pseudopotential for potassium in the lowest three angular momentum channels. We plot the sum of the pseudopotential and the centrifugal term $\ell(\ell+1) / 2 r^{2}$. The corresponding hydrogen potentials are shown for each channel. For distances greater than about 5 au the two potentials agree, which means that $V_{c}$ and $V_{\text {pol }}$ have become negligible.

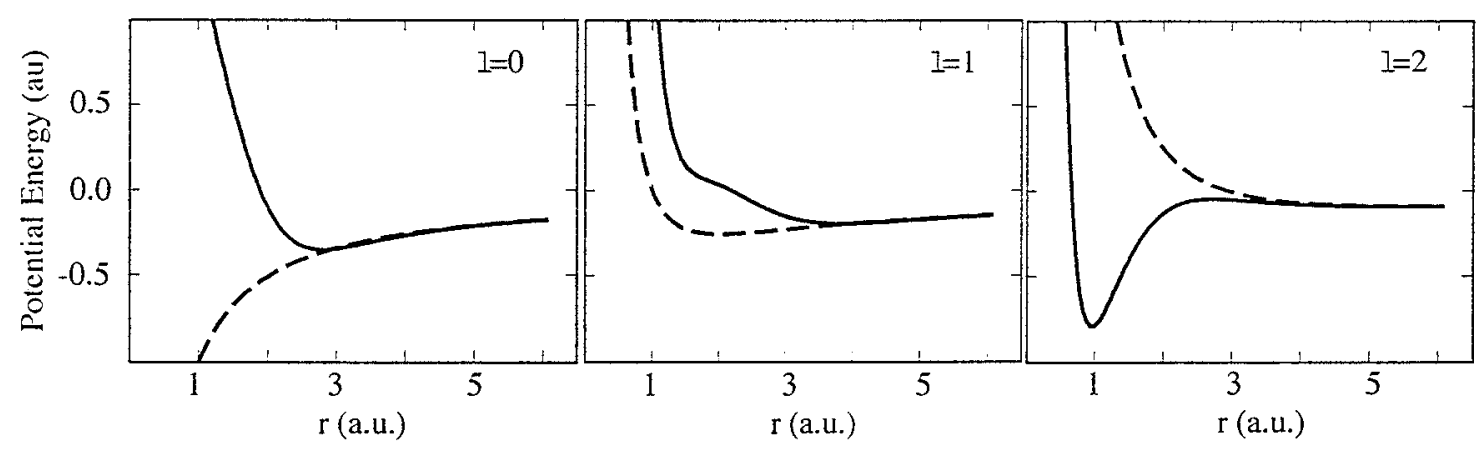

FIGURE 1. Solid line: potassium potential for $\ell=0-2$. We plot the sum of the pseudopotential and the centrifugal term. The corresponding potential for hydrogen is shown as a dashed line. 


\section{Mixed Gauge Propagation}

The choice of electromagnetic gauge has a large influence on the numerical effort involved in strong field calculations, through both the number of spherical harmonics $\ell_{\max }$ and the time step $\delta t$ required for convergence [10]. In the length gauge the TDSE in the dipole approximation for an electron in a time-varying field polarized along the $z$ axis is

$$
i \frac{\partial \psi_{l}}{\partial t}=\left(H_{0}+\mathcal{E}(t) z\right) \psi_{l}
$$

where $H_{0}$ is the field-free ion-valence electron Hamiltonian and $\mathcal{E}(t)$ is the MIR laser electric field.

This gauge is preferred near the ion core where the interaction is reasonably small, even for intense fields. When the electron is ionized, however, it travels far from the ion core where the interaction grows rapidly. This has two undesirable consequences. First, the large interaction requires that a small time step be used in the time integration. Second, the oscillations in the electron's motion induced by the field, which are on the order of the free electron oscillation amplitude $\sim \mathcal{E} / \omega^{2}$ [11], require the use of a very large number of angular momentum functions.

If we define the vector potential $A(t)$ and an auxiliary function $\Phi(t)$ by the relations

$$
-\frac{\partial A(t)}{\partial t}=\mathcal{E}(t) ; \quad \frac{\partial \Phi}{\partial t}=\frac{1}{2} A^{2}(t),
$$

then for local potentials we can use the gauge transformation

$$
\psi_{l}(\vec{r}, t)=e^{-i(A(t) z+\Phi(t))} \psi_{v}(\vec{r}, t)
$$

to write the TDSE is the velocity gauge as

$$
i \frac{\partial \psi_{v}}{\partial t}=\left(H_{0}-i A(t) \frac{\partial}{\partial z}\right) \psi_{v} .
$$

In this gauge the phase factor that describes the free-electron like oscillations in the ionized electron's wave function is mostly removed, which results in a large reduction in the number of angular momentum channels needed for convergence. The interaction is also bounded when the electron travels far from the ion core. Close to the ion core, however, the interaction $A(t) p_{z}$ can be very large which again makes the equations hard to integrate. The two interactions are comparable in strength at a distance from the ion core on the order of the free electron oscillation amplitude.

We have developed a mixed gauge time propagation technique for solving the TDSE which uses the length gauge near the ion core and the velocity gauge at large distances. We switch gauges in a transition zone that is located away from the ion 
core. This insures that the potential is strictly local in the transition zone (the short range $\ell$-dependent terms having decayed) and the local gauge transformation Eq. 5 can be used. The mixed gauge approach allows us to use non-local pseudopotentials near the ion core, which give a superior description of the atomic potentials as compared to local pseudopotentials, while minimizing the numerical effort.

To implement the mixed gauge time propagation we use two partially overlapping radial grids. The length gauge grid extends from $r=0$ to $r_{l}$ and the velocity gauge grid extends from $r_{v}$ to $R$, which is the maximum grid radius. The grids overlap, $r_{v}<r_{l}$, and the midpoint of the transition zone is $r_{0}$. At each time step $t_{n}$ we propagate both wave functions forward in time to $t_{n+1}=t_{n}+\delta t$ on their separate grids. Due to the presence of the artificial boundaries, some error accumulates in $\psi_{l}$ near $r_{l}$ and in $\psi_{v}$ near $r_{v}$. Before taking the next propagation step we replace the "bad" part of the wave function on each grid with "good" wave function that we obtain by using the gauge transformation on the wave function from the other grid. For instance, after every time step, the portion of $\psi_{l}$ between $r_{0}$ and $r_{l}$ is replaced by the gauge-transformed $\psi_{v}$ at the same radial grid points. Typically, $r_{l}=30$ au

and $r_{v}=20 \mathrm{au}$. The key to this method is that the errors at each propagation step are localized near the artificial boundaries. This is due to the fact that we use a finite difference discretization of the TDSE. We turn next to a discussion of these finite difference equations.

\section{Discretizing the TDSE}

In this section we derive a discretized form of the time-dependent Schrödinger equation in both the length and velocity gauges. The length gauge discretization has been discussed before in somewhat less detail in [6]. We use a uniform finite difference grid, however, non-uniform grid versions of these equations have also been used in the study of Rydberg wave packets [12]. Instead of discretizing the TDSE directly, we begin by discretizing the action $[6,13,14]$, defined as

$$
\mathcal{S}=\int_{t_{1}}^{t_{2}} d t \mathcal{L}\left(\psi, \psi^{*}\right)
$$

where the Lagrangian is

$$
\mathcal{L}=\left\langle\psi\left|i \frac{\partial}{\partial t}-T-V-H_{I}\right| \psi\right\rangle .
$$

$H_{I}$ is the electron-laser interaction in either gauge. We include the centrifugal term $\ell(\ell+1) / 2 r^{2}$ in $V$, so that $T$, referred to as $T_{r}$ in what follows, is the radial kinetic energy operator. Variation of the action with respect to $\psi^{*}, \frac{\delta S}{\delta \psi^{*}}=0$, for fixed $t_{1}$ and $t_{2}$, yields an equation of motion for $\psi$. This is equivalent to requiring that $\psi^{*}$ obey the Euler-Lagrange condition

$$
\frac{d}{d t}\left(\frac{\partial \mathcal{L}}{\partial \dot{\psi}^{*}}\right)-\frac{\partial \mathcal{L}}{\partial \psi^{*}}=0 .
$$


For a continuous wave function this procedure leads directly to the TDSE for $\psi$. For a discretized wave function, the procedure leads to a discretized version of the TDSE that efficiently accounts for the boundary conditions imposed on $\psi$, especially at small $r$.

The radial dimension $r_{\min }=0$ to $r_{\max }=R$ is divided into $N_{r}$ equal intervals with knots at $r_{j}=(j-1 / 2) \Delta r\left\{j=1 \ldots N_{r}\right\}$. The wave function is known at these points only. Typically, $\Delta r=0.25$ au. We assume that $\psi_{\ell}(R)=0$, as well as $r \psi_{\ell}(r)=0$, and $r \frac{\partial \psi_{\ell}}{\partial r}=0$ at $r=0$. With these boundary conditions, we can derive equations of motions for the coefficients $\psi_{\ell}^{j} \equiv \psi_{\ell}\left(r_{j}, t\right)$.

We begin by choosing approximate integration schemes for the expectation values in the Lagrangian which are second order in the grid spacing $\Delta r$. This will lead to finite difference equations that are accurate to the same order.[13]. After integrating over the angular variables, the remaining integrals in $\mathcal{L}$ are discretized as

$$
\begin{aligned}
\left\langle i \frac{\partial}{\partial t}\right\rangle & =\sum_{\ell=0}^{\ell_{\max }} \int_{0}^{R} d r r^{2} \psi_{\ell}^{*} i \frac{\partial \psi_{\ell}}{\partial t} \approx \Delta r \sum_{\ell=0}^{\ell_{\max }} \sum_{j=1}^{N_{r}} r_{j}^{2}\left(\psi_{\ell}^{j}\right)^{*} i \frac{\partial \psi_{\ell}^{j}}{\partial t} \\
\left\langle T_{r}\right\rangle & =\frac{1}{2} \sum_{\ell=0}^{\ell_{\max }} \int_{0}^{R} d r r^{2}\left|\frac{\partial \psi_{\ell}}{\partial r}\right|^{2} \approx \frac{\Delta r}{2} \sum_{\ell=0}^{\ell_{\max }} \sum_{j=1}^{N_{r}} r_{j+1 / 2}^{2}\left|\frac{\psi_{\ell}^{j+1}-\psi_{\ell}^{j}}{\Delta r}\right|^{2} \\
\langle V\rangle & =\sum_{\ell=0}^{\ell_{\max }} \int_{0}^{R} d r r^{2} \psi_{\ell}^{*} V_{\ell}(r) \psi_{\ell} \approx \Delta r \sum_{\ell=0}^{\ell_{\max }} \sum_{j=1}^{N_{r}} r_{j}^{2}\left(\psi_{\ell}^{j}\right)^{*} V_{\ell}^{j} \psi_{\ell}^{j}
\end{aligned}
$$

As can be seen from these expressions, the derivative term is evaluated at the mid point of each interval, and the potential term is evaluated at the grid points. The length gauge interaction term is

$$
\begin{aligned}
\mathcal{E}(t)\langle z\rangle & =\sum_{\ell=0}^{\ell_{\max }} \int_{0}^{R} d r r^{2} \phi_{\ell}^{*} \mathcal{E}(t) r\left[c_{\ell} \psi_{\ell+1}+c_{\ell-1} \psi_{\ell-1}\right] \\
& \approx \Delta r \sum_{\ell=0}^{\ell_{\max }} \sum_{j=1}^{N_{r}} r_{j}^{2}\left(\psi_{\ell}^{j}\right)^{*} \mathcal{E}(t) r_{j}\left[c_{\ell} \psi_{\ell+1}^{j}+c_{\ell-1} \psi_{\ell-1}^{j}\right] .
\end{aligned}
$$

where we have made use of

$$
z|\ell\rangle=r\left[c_{\ell}|\ell+1\rangle+c_{\ell-1}|\ell-1\rangle\right]
$$

$c_{\ell}$ is the $3 j$ coefficient $c_{\ell}=\frac{\ell+1}{\sqrt{(2 \ell+1)(2 \ell+3)}}$ (note that $c_{-1}=0$ ).

Substituting these expressions into $\mathcal{L}$ and shifting indices on the radial summations in accord with the boundary conditions stated above, leads to

$$
\begin{aligned}
\mathcal{L}=\Delta r \sum_{\ell=0}^{\ell_{\max }} \sum_{j=1}^{N_{r}} r_{j}^{2}\left(\psi_{\ell}^{j}\right)^{*} & \left\{i \frac{\partial \psi_{\ell}^{j}}{\partial t}+\frac{1}{2(\Delta r)^{2}}\left[\left(\psi_{\ell}^{j+1}-\psi_{\ell}^{j}\right) \frac{r_{j+1 / 2}^{2}}{r_{j}^{2}}-\left(\psi_{\ell}^{j}-\psi_{\ell}^{j-1}\right) \frac{r_{j-1 / 2}^{2}}{r_{j}^{2}}\right]\right. \\
& \left.-V_{\ell}^{j} \psi_{\ell}^{j}-\mathcal{E}(t) r_{j}\left[c_{\ell} \psi_{\ell+1}^{j}+c_{\ell-1} \psi_{\ell-1}^{j}\right]\right\} .
\end{aligned}
$$


We make a transformation to the scaled coefficients $\phi_{j}^{\ell}$ which are related to $\psi_{\ell}^{j}$ by

$$
\phi_{\ell}^{j}=r_{j} \psi_{\ell}^{j}
$$

These satisfy the normalization condition

$$
\Delta r \sum_{\ell=0}^{\ell_{\max }} \sum_{j=1}^{N_{r}}\left|\phi_{\ell}^{j}\right|^{2}=1
$$

Imposing the Euler-Lagrange condition with respect to $\left(\phi_{\ell}^{j}\right)^{*}$, we arrive at an equation for the time evolution of $\phi_{\ell}^{j}$. This equation is

$$
i \frac{\partial \phi_{\ell}^{j}}{\partial t}=\left[\hat{H}_{0} \phi\right]_{\ell}^{j}+\left[\hat{H}_{I}^{l} \phi\right]_{\ell}^{j}
$$

where

$$
\begin{aligned}
& {\left[\hat{H}_{0} O\right]_{\ell}^{j}=-\frac{1}{2(\Delta r)^{2}}\left[\alpha_{j} \phi_{\ell}^{j+1}-2 \beta_{j} \phi_{\ell}^{j}+\alpha_{j-1} \phi_{\ell}^{j-1}\right]+V_{\ell}^{j} \phi_{\ell}^{j}} \\
& {\left[\hat{H}_{I}^{l} O\right]_{\ell}^{j}=\mathcal{E}(t) r_{j}\left[c_{\ell} \phi_{\ell+1}^{j}+c_{\ell-1} \phi_{\ell-1}^{j}\right] .}
\end{aligned}
$$

The dimensionless coefficients $\alpha_{j}$ and $\beta_{j}$ are

$$
\alpha_{j}=\frac{r_{j-1 / 2}^{2}}{r_{j}^{2}}=\frac{j^{2}}{j^{2}-1 / 4} ; \quad \beta_{j}=\frac{r_{j+1 / 2}^{2}+r_{j-1 / 2}^{2}}{2 r_{j}^{2}}=\frac{j^{2}-j+1 / 2}{j^{2}-j+1 / 4} .
$$

$\hat{H}_{0}$ is the field-free Hamiltonian matrix, which is diagonal in the $\ell$ quantum number and tridiagonal in the radial index $j$. Likewise, $\hat{H}_{I}^{l}$ is diagonal in $j$ and tridiagonal in $\ell$. Far from the $r=0$ boundary both $\alpha_{j}$ and $\beta_{j}$ approach 1 , and we recover the standard second order finite difference equations.

In the velocity gauge, the interaction term is more complicated since it couples both the radial and the angular coordinates [15]:

$$
\frac{\partial}{\partial z}|\ell\rangle=c_{\ell}\left(\frac{\partial}{\partial r}-\frac{\ell}{r}\right)|\ell+1\rangle+c_{\ell-1}\left(\frac{\partial}{\partial r}+\frac{\ell+1}{r}\right)|\ell-1\rangle .
$$

The expectation value which enters the Lagrangian is

$$
i A(t)\left\langle\frac{\partial}{\partial z}\right\rangle=i . A(t) \sum_{\ell=0}^{\ell_{\max }} \int r^{2} d r\left[c_{\ell} \psi_{\ell+1}^{*}\left(\frac{\partial \psi_{\ell}}{\partial r}-\frac{\ell}{r} \psi_{\ell}\right)+c_{\ell-1} \psi_{\ell-1}^{*}\left(\frac{\partial \psi_{\ell}}{\partial r}+\frac{\ell+1}{r} \psi_{\ell}\right)\right]
$$

Straightforward discretization of this expression does not yield a Hermitian matrix so we first integrate the $\psi_{\ell-1}^{*} \frac{\partial \psi_{\ell}}{\partial r}$ term by parts and rearrange the $\ell$ indices to get an equivalent expression: 


$$
i A(t)\left\langle\frac{\partial}{\partial z}\right\rangle=i A(t) \sum_{\ell=0}^{\ell_{\max }} \int r^{2} d r\left[c_{\ell-1} \psi_{\ell}^{*}\left(\frac{\partial}{\partial r}-\frac{\ell-1}{r}\right) \psi_{\ell-1}-c_{\ell}\left(\frac{\partial \psi_{\ell}^{*}}{\partial r}+\psi_{\ell}^{*} \frac{\ell}{r}\right) \psi_{\ell+1}\right]
$$

We write the resulting discretized interaction Hamiltonian as the sum of two terms

$$
\begin{gathered}
{\left[\hat{H}_{I}^{v} \phi\right]_{\ell}^{j}=\left[\hat{H}_{1}^{v} \phi\right]_{\ell}^{j}+\left[\hat{H}_{2}^{v} \phi\right]_{\ell}^{j}} \\
{\left[\hat{H}_{1}^{v} \phi\right]_{\ell}^{j}=\frac{i A(t)}{r_{j}}\left[(\ell+1) c_{\ell} \phi_{\ell+1}^{j}-\ell c_{\ell-1} \phi_{\ell-1}^{j}\right]} \\
{\left[\hat{H}_{2}^{v} \phi\right]_{\ell}^{j}=\frac{i A(t)}{2 \Delta r}\left[c_{\ell}\left(\alpha_{j} \phi_{\ell+1}^{j+1}-\alpha_{j-1} \phi_{\ell+1}^{j-1}\right)+c_{\ell-1}\left(\alpha_{j} \phi_{\ell-1}^{j+1}-\alpha_{j-1} \phi_{\ell-1}^{j-1}\right)\right]}
\end{gathered}
$$

The first term, weighted by $1 / r_{j}$, is tridiagonal in the $\ell$ coordinate. The second term couples the radial and angular coordinates, and is tridiagonal in both.

\section{Time Propagation}

The Hamiltonian in the both the length and velocity gauges consists of two pieces, $\hat{H}_{0}$, the atomic Hamiltonian, and the interaction piece $\hat{H}_{I}$. In both gauges we use a split-operator expansion of the full short-time propagator which is unitary and correct to $\mathcal{O}(\delta t)^{3}$.

$$
\begin{aligned}
\psi(t+\delta t) & =e^{-i \hat{H}(t) \delta t} \psi(t) \\
& =e^{-i\left(\hat{H}_{0}+\hat{H}_{I}(t)\right) \delta t} \psi(t) \approx e^{-i \hat{H}_{I}(t) \delta t / 2} e^{-i \hat{H}_{0} \delta t} e^{-i \hat{H}_{I}(t) \delta t / 2} \psi(t)
\end{aligned}
$$

The interaction term is evaluated at the midpoint of the time step. The action of the exponentials on the time-dependent wave function can not be calculated directly due to the non-diagonal nature of the matrices representing $\hat{H}_{0}$ and $\hat{H}_{I}$. We therefore resort to approximations to the full exponentials which are themselves unitary and correct to the same order in $\delta t$ as the split-operator method. The propagator for $\hat{H}_{0}$ is approximated by the Crank-Nicholson form [13]

$$
e^{-i \hat{H}_{0} \delta t} \approx\left[1+i \hat{H}_{0} \delta t / 2\right]^{-1}\left[1-i \hat{H}_{0} \delta t / 2\right]
$$

The interaction propagator is handled using a $2 \times 2$ splitting method previously used by Richardson and others [16] for cartesian grids. We illustrate this first in the length gauge. In the length gauge, $\hat{H}_{I}^{l}$ is diagonal in $j$ and we can treat the wave function at each radial point separately. For each radial index $j$, the matrix that must be exponentiated, $\hat{L}^{j}$, is tridiagonal in the $\ell$ index.

$$
e^{-i \hat{H}_{I} \delta t / 2} \phi=e^{-i \hat{L}^{j}} \phi^{j}
$$

$\hat{L}^{j}$ can be split into even and odd pieces $\hat{L}^{j}=\hat{L}^{e}+\hat{L}^{o}$ as shown below: 


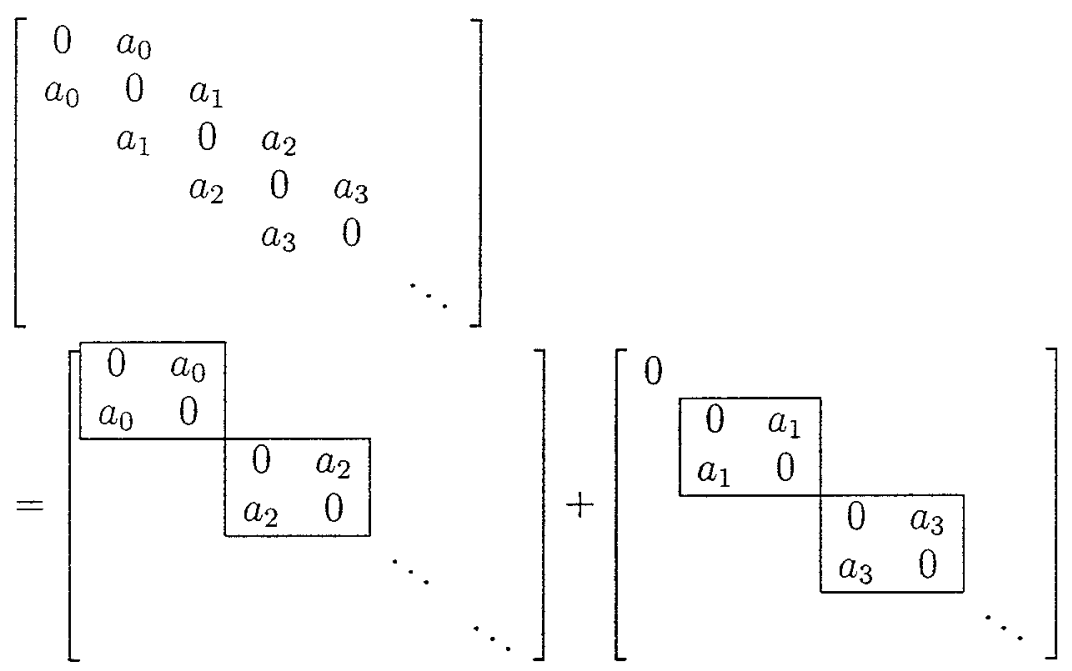

Where the matrix elements are $a_{\ell}=\mathcal{E}(t) c_{\ell} r_{j} \delta t / 2$. The even and odd matrices consist of $2 \times 2$ block diagonal pieces which are easily exponentiated using

$$
\exp \left\{-i\left[\begin{array}{ll}
0 & a \\
a & 0
\end{array}\right]\right\}=\left[\begin{array}{rr}
\cos a & -i \sin a \\
-i \sin a & \cos a
\end{array}\right]
$$

A single propagation step in the length gauge is accomplished via

$$
e^{-i \hat{H}^{l} \delta t}=e^{-i \hat{L}^{e} \delta t / 2} e^{-i \hat{L}^{o} \delta t / 2}\left[1+i \hat{H}_{0} \delta t / 2\right]^{-1}\left[1-i \hat{H}_{0} \delta t / 2\right] e^{-i \hat{L}^{o} \delta t / 2} e^{-i \hat{L}^{e} \delta t / 2}
$$

Although the matrices $\hat{L}^{e}$ and $\hat{L}^{o}$ do not commute, the symmetric placement of the operators makes this approximate propagator unitary and correct to $\mathcal{O}(\delta t)^{3}$.

Propagation in the velocity gauge is handled in a very similar manner. Referring to Eq. 21, we use the symmetric splitting

$$
e^{-i \hat{H}^{v} \delta t} \approx e^{-i \hat{H}_{2} \delta t / 2} e^{-i \hat{H}_{1} \delta t / 2}\left[1+i \hat{H}_{0} \delta t / 2\right]^{-1}\left[1-i \hat{H}_{0} \delta t / 2\right] e^{-i \hat{H}_{1} \delta t / 2} e^{-i \hat{H}_{2} \delta t / 2}
$$

The terms involving $\hat{H}_{1}$ (Eq. 22) are of the same form as the interaction term in the length gauge and are treated in the same way.

$$
e^{-i \hat{H}_{1} \delta t / 2} \phi=e^{\hat{M}^{j}} \phi^{j}
$$

where the non-zero elements of the antisymmetric matrix of $\hat{M}^{j}$ are $a_{\ell}=A(t) r_{j}^{-1}(\ell+1) c_{\ell} \delta t / 2$. Again the full matrix is split into even and odd $2 \times 2$ block-diagonal matrices and the exponentiation is accomplished using the relation

$$
\exp \left\{\left[\begin{array}{rr}
0 & a \\
-a & 0
\end{array}\right]\right\}=\left[\begin{array}{rr}
\cos a & \sin a \\
-\sin a & \cos a
\end{array}\right] .
$$

Exponentiating the terms involving $\hat{H}_{2}$ (Eq. 23) is more complicated since they couple $\phi_{\ell}^{j}$ to the four adjacent coefficients $\phi_{\ell \pm 1}^{j \pm 1}$. To do it, we use the $2 \times 2$ splitting 
method twice in succession, first in the $\ell$ dimension and then in the $j$ dimension. The exponent $-i \hat{H}_{2} \delta t / 2$ can be written as a super-matrix, $\widetilde{N}$, which is tridiagonal in $\ell$ :

$$
\widetilde{N}=-i \hat{H}_{2} \delta t / 2=\left[\begin{array}{cccc}
0 & \hat{a}_{0} & 0 & \\
\hat{a}_{0} & 0 & \hat{a}_{1} & \\
0 & \hat{a}_{1} & 0 & \\
& & & \ddots
\end{array}\right] .
$$

Each of the elements $\hat{a}_{\ell}$ are themselves matrices that are tridiagonal in $j$ :

$$
\hat{a}_{\ell}=c_{\ell} \frac{A(t)}{2 \Delta r} \frac{\delta t}{2}\left[\begin{array}{cccc}
0 & \alpha_{1} & 0 & \\
-\alpha_{1} & 0 & \alpha_{2} & \\
0 & -\alpha_{2} & 0 & \\
& & & \ddots
\end{array}\right]
$$

with $\alpha_{j}$ given by Eq. 20. We now split the super-matrix $\widetilde{N}$ into even and odd pieces which consist of $2 \times 2$ blocks just as in Eq. 28 .

$$
e^{\tilde{N}}=e^{\tilde{N}^{e}} e^{\tilde{N}^{o}}
$$

Before applying each of these exponentials, we diagonalize using a similarity transformation:

$$
\frac{1}{\sqrt{2}}\left[\begin{array}{rr}
1 & 1 \\
1 & -1
\end{array}\right] \exp \left\{\left[\begin{array}{rr}
0 & \hat{a}_{\ell} \\
\hat{a}_{\ell} & 0
\end{array}\right]\right\}\left[\begin{array}{rr}
1 & 1 \\
1 & -1
\end{array}\right] \frac{1}{\sqrt{2}}=\left[\begin{array}{cc}
e^{\hat{a}_{\ell}} & 0 \\
0 & e^{-\hat{a}_{\ell}}
\end{array}\right] .
$$

The application of the operators $e^{ \pm \hat{a}_{\ell}}$ is then made by further splitting the matrices $\hat{a}_{\ell}$ into even and odd pieces in the $j$ index as in Eq. 28, and then using Eq. 31. The various even and odd matrices are placed symmetrically in Eq. 29 so that it remains unitary.

Both the length and velocity gauge propagators are unitary and unconditionally stable. All of the various operations scale as $N_{r} \times \ell_{\max }$, which means that the overall numerical effort involved in using our multiple gauge approach is linear in the total number of grid points. We can determine about $1.2 \times 10^{6}$ space-time points per CPU second on a Cray Y/MP.

\section{RESULTS}

In this section we discuss some illustrative results from our calculations of photoelectron and photoemission spectra from alkali metal atoms in MIR fields. Further details of the theoretical results, and their connection to experiments on alkali metal atoms, can be found in references $[5,7,8]$. 


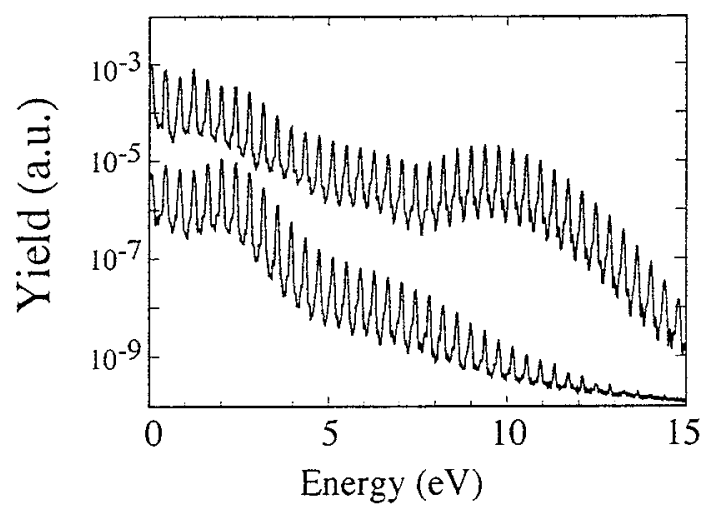

FIGURE 2. ATI spectra for potassium (upper curve) and sodium (lower curve) at $10^{12} \mathrm{~W} / \mathrm{cm}^{2}$ and $3.2 \mu \mathrm{m}$. The ponderomotive energy is $1 \mathrm{eV}$.

In Fig. 2 we show total (angle integrated) ATI spectra for potassium and sodium, at a wavelength of $3.2 \mu \mathrm{m}$, corresponding to a photon energy of $0.39 \mathrm{eV}$. Ionization of $\mathrm{K}$ requires a minimum of 11 photons at this wavelength. The laser pulse has a trapezoidal shape, consisting of one cycle ramps and five cycles of constant intensity. The spectrum is calculated using an energy window function [17]. The peak intensity is $10^{12} \mathrm{~W} / \mathrm{cm}^{2}$, which corresponds to a ponderomotive energy of about $1 \mathrm{eV}$. The difference in the ionization yields is due to the higher ionization potential of sodium as compared with potassium. These results are representative of calculations over a range of laser intensities. The calculation used $\ell_{\max }=16$ and $\delta t=0.1$ au. Calculations using the length gauge for the entire radial grid required $\ell_{\max }=200$ and $\delta t=.02$ au for a similar level of convergence.

Fig. 2 illustrates a striking phenomena in the alkali metals. The production of high order photoelectrons is very sensitive to the atomic species. $K$ exhibits a plateau extending up to the about $10 U_{p}$, whereas the $\mathrm{Na}$ distribution shows essentially no change of slope beyond $3 U_{p}$. Fig. 3 shows that this difference persists over a range of intensities. Indeed, Na never shows any significant high-order ATI production while $\mathrm{K}$ shows an efficiency that is higher than that observed in the rare gases [18]. Since the high energy portion of the ATI spectrum results from the rescattering of the electron from the ion core after it has been initially freed and accelerated in the strong field [18-21], it is reasonable to try to connect this difference in rescattering efficiency to the electron-ion potential. Our calculations show that the very efficient rescattering in $K$ is mostly due to the attractive short range part of the $\ell=2$ potential. This attractive piece is absent in Na. More details of these results, as well as experimental confirmation of the predictions implicit in Figs. 2-3, can be found in the paper by Sheehy, et al. in this volume and in [7].

One of the most compelling reasons to study strong field processes in the MIR is the possibility of generating high order harmonics in the UV/visible portion of the spectrum $[5,21]$. Potentially these harmonics could be completely characterized (both amplitude and phase) using conventional techniques. This would allow for a deeper understanding of HHG in general, and may provide insight into the 


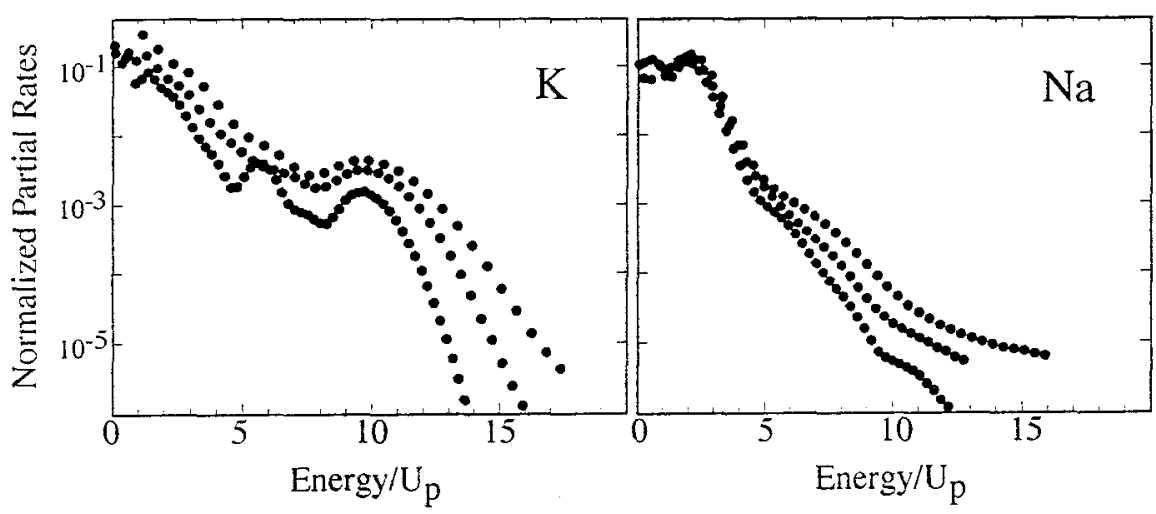

FIGURE 3. Normalized partial rates (ionization rate per ATI peak) for $\mathrm{K}$ and $\mathrm{Na}$ for three intensities between 0.75 and $1.5 \mathrm{TW} / \mathrm{cm}^{2}$. The individual points have been scaled so that the sum of the points for each data set is equal to one. The lowest intensity data set is the top one for each atom. Note that the energy has been scaled by $U_{p}$

question of whether the XUV harmonics generated from the rare gases can be used to produce attosecond pulses. A discussion of the first experimental observation of HHG in alkali metal atoms ( $\mathrm{K}$ and $\mathrm{Rb}$ ) can be found in references [5] and [8].

Fig. 4 shows a single-atom harmonic spectrum calculated for a potassium atom in a $3.2 \mu \mathrm{m}, 80$ cycle laser pulse with a peak intensity of $1 \mathrm{TW} / \mathrm{cm}^{2}$. The spectrum exhibits many similarities with the better-known near-IR excitation of rare gas atoms, especially the general form of the spectrum and the cut off. However, as mentioned above, the alkali metals present several interesting and potentially useful contrasts to the rare gases. Chief among these is the strong coupling between the $n s$ ground state and the first excited $n p$ state.

As an example of the role played by this strong coupling, we compare the full result, shown in white squares, to two approximate calculations. The approximate calculations start by defining a subspace projection operator $\hat{P}_{n}$ :

$$
\hat{P}_{n}=|1\rangle\langle 1|+| 2\rangle\langle 2|+\ldots| n\rangle\langle n| .
$$

The projected wave function at a time $t$ is $\left|\Phi_{n}(t)\right\rangle=\hat{P}_{n}|\psi(t)\rangle$, where $|\psi(t)\rangle$ is the full time-dependent wave function. The approximate dipole moment is calculated by requiring that the transition either begin or end in the projected subspace:

$$
\langle d\rangle(t)=\langle\psi(t)|z| \psi(t)\rangle \approx\left\langle\psi(t)\left|\hat{P}_{n} z+z \hat{P}_{n}\right| \psi(t)\right\rangle-\left\langle\psi(t)\left|\hat{P}_{n} z \hat{P}_{n}\right| \psi(t)\right\rangle .
$$

As $n \rightarrow \infty$ and $\hat{P}_{n} \rightarrow 1$, the approximate dipole goes over to the full dipole, as it should. The last term makes up for double counting of transitions between states in the projected subspace. An equivalent way to write the approximate dipole that makes this clear is

$$
\langle d\rangle(t) \approx\left\langle d_{n}\right\rangle(t)=\left\langle\psi|z| \Phi_{n}\right\rangle+\left\langle\Phi_{n}|z| \psi\right\rangle-\left\langle\Phi_{n}|z| \Phi_{n}\right\rangle .
$$

This applies equally well when we calculate the acceleration form of the dipole. 


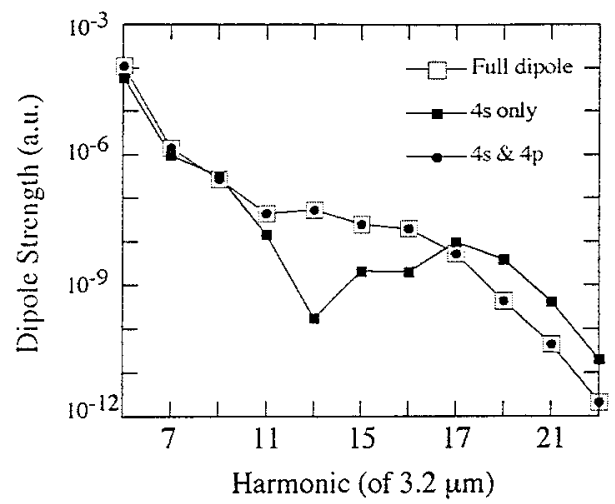

FIGURE 4. Single atom harmonic spectrum for K. The full calculation is compared to approximate dipoles calculated using the continuum wave packet plus the $4 s$ and the $4 s+4 p$ states.

For potassium, which has a $4 s$ ground state, we compare approximate dipoles calculated using

$$
\hat{P}_{1}=|4 s\rangle\langle 4 s|
$$

and

$$
\hat{P}_{2}=|4 s\rangle\langle 4 s|+| 4 p\rangle\langle 4 p|
$$

The black squares in Fig. 4 are the result of including the dipole interaction between the time-dependent wave function and the ground (4s) state. This approximation, which works very well in the rare gases, fails here, showing differences in both the conversion efficiency and the harmonic cutoff energy. However, when we include the first excited state in the projected subspace, as shown with black circles (inside the white squares), we get perfect agreement with the full result.

The important role played by the coupling of the first excited $n p$ state in $\mathrm{HHG}$ in the alkali metals provides an interesting and potentially useful contrast with HHG from rare gases. For example, it might be possible to achieve control over the HHG process by using a second laser field to alter either the $n s \rightarrow n p$ or the $n p \rightarrow$ continuum coupling. This is just one example of the potential new applications that may result from extending strong field multiphoton studies to the mid-infrared wavelength regime.

\section{ACKNOWLEDGMENTS}

K. J. S. and M. B. G. acknowledge support from the National Science Foundation through grant No. PHY-9733890, the Louisiana State Board of Regents through grant No. LEQSF96-99-RD-A-14, and the Swedish National Science Research Council. Computer time was provided by the National Supercomputer Centre in Sweden. This research was carried out in part under the auspices of the 
U. S. Department of Energy at Lawrence Livermore National Laboratory under contract No. W-7405-ENG-48, and in part at Brookhaven National Laboratory under contract No. DE-AC02-98CH10886, with the support of its Division of Chemical Sciences, Office of Basic Energy Sciences and BNL/LDRD No. 99-56.

\section{REFERENCES}

1. B. Sheehy and L. F. DiMauro, Ann. Rev. Phys. Chem. 47, 463 (1996).

2. B. Sheehy, J. D. D. Martin and L. F. DiMauro, J. Opt. Soc. Am. B submitted (1999).

3. L. V. Keldysh, Sov. Phys. JETP 20, 1307 (1965).

4. $U_{p}$ measured in $\mathrm{eV}$ is given by $0.093 I \lambda^{2}$ where $I$ is the intensity in $\mathrm{TW} / \mathrm{cm}^{2}$ and $\lambda$ is the wavelength in microns. The long wavelength, high intensity limit is defined by $U_{p}$ being substantially larger than the photon energy. For $1 \mathrm{TW} / \mathrm{cm}^{2}, 3.2 \mu \mathrm{m}$ light $U_{p}$ is $1 \mathrm{eV}$, which corresponds to about three MIR photons.

5. B. Sheehy, J. D. D. Martin, L. F. DiMauro, P. Agostini, K. J. Schafer, M. B. Gaarde, and K. C. Kulander, Phys. Rev. Lett. 83, 5270 (1999).

6. K. C. Kulander, K. J. Schafer, and J. L. Krause, in Atoms in Intense Radiation Fields, Ed. M. Gavrila (Academic Press, New York, 1992).

7. M. B. Gaarde, K. J. Schafer, K. C. Kulander, B. Sheehy, D. W. Kim, and L. F. DiMauro, Phys. Rev. Lett. submitted (1999).

8. B. Sheehy, J. D. D. Martin, T. Clatterbuck, D. W. Kim, L. F. DiMauro, P. Agostini, K. J. Schafer, M. B. Gaarde, and K. C. Kulander, this volume.

9. W. J. Stevens et al, Can. J. Chem. 70, 612 (1992).

10. E. Cormier and P. Lambropoulos, J. Phys. B 29, 1667 (1996); H. G. Muller, Laser Physics 9, 138 (1999).

11. The free electron oscillation amplitude measured in atomic units is given by $2.6 \sqrt{I} \lambda^{2}$, where $I$ is the intensity in $\mathrm{TW} / \mathrm{cm}^{2}$ and $\lambda$ is the wavelength in microns. For 1 $\mathrm{TW} / \mathrm{cm}^{2}, 3.2 \mu \mathrm{m}$ light the amplitude is $\approx 26 \mathrm{au}$.

12. J. L. Krause and K. J. Schafer, J. Phys. Chem. A 10310118 (1999).

13. S. E. Koonin, Computational Physics, Menlo Park, CA: Benjamin/Cummings, Inc., 1986.

14. J. Negele, Rev. Mod. Phys. 54913 (1982).

15. J. L. Krause, J. D. Morgan, and R. S. Berry, Phys. Rev. A 353189 (1987).

16. J. L. Richardson, Comp. Phys. Comm. 6384 (1991); H. DeRaedt, Comp. Phys. Report 71 (1987).

17. K. J. Schafer Comp. Phys. Comm. 63427 (1991).

18. G. G. Paulus et al, Phys. Rev. Lett. 72, 2851 (1994).

19. K. J. Schafer et al, Phys. Rev. Lett. 70, 1599 (1993).

20. P. B. Corkum, Phys. Rev. Lett. 71, 1994 (1993).

21. B. Walker et al, Phys. Rev. Lett. 77, 5031 (1996). 


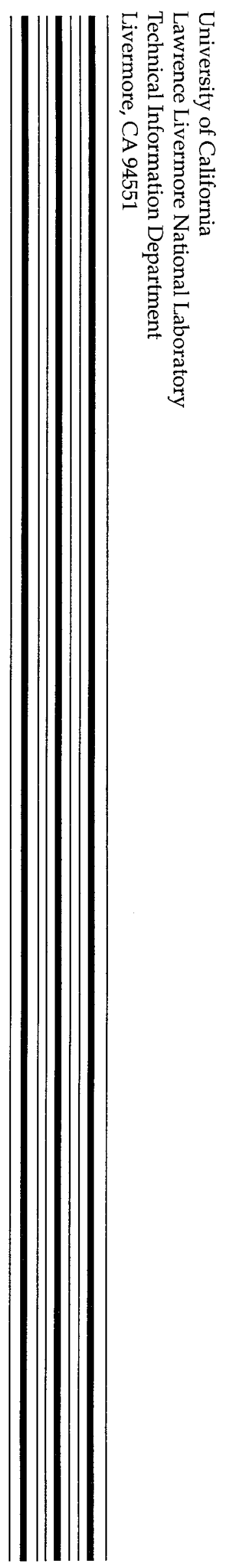

